\title{
A huge thrombus trapped in the patent foramen ovale
}
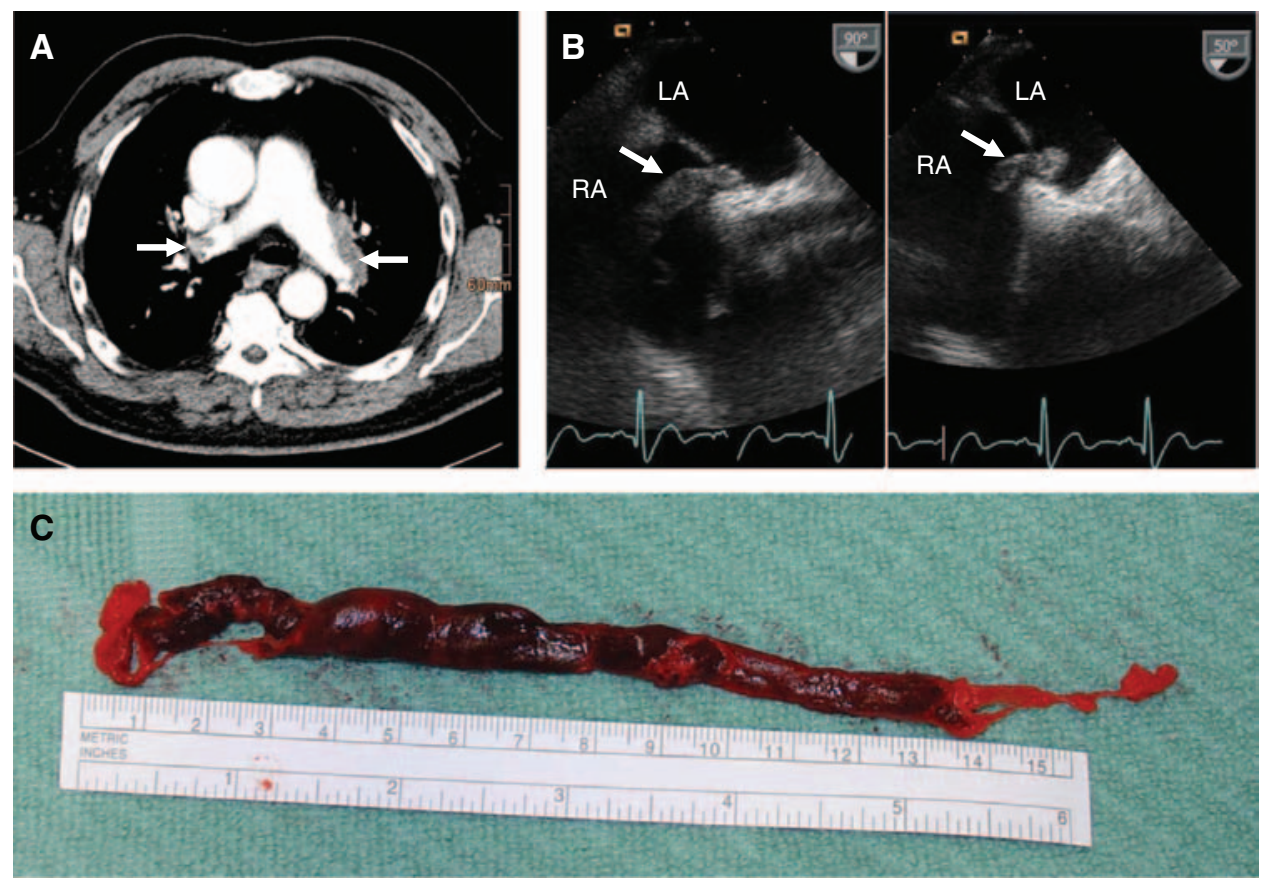

A 62-year-old man with a history of a lumbar disc prolapse and concomitant immobilization for about 8 weeks was admitted to a district hospital because of increasing shortness of breath. During that time no treatment with low molecular heparin was administered. Furthermore, sweating and tachycardia were noted. Chest computed tomography showed extensive central pulmonary embolism (arrows) with an enlarged right heart (Panel A).

Ultrasound investigation revealed deep venous thrombosis of the right lower limb.

Transthoracic echocardiography showed normal left ventricular ejection fraction and right atrial and ventricular distension with a floating mass in the right atrium (RA). The patient was then referred to our service for further work-up.

Transthoracal echocardiograpy confirmed the floating mass in the right atrium and also raised the suspicion of a floating mass in the left atrium. Transesophageal echocardiograpy subsequently performed depicted a huge thrombus (arrow) crossing not only the tricuspid valve but also the foramen ovale into the left atrium (LA) (Panel B) partly crossing the mitral valve. Clinical history and screening tests for thrombophilia were negative. Due to the compromised respiratory situation, right heart overload, and the above-described huge thrombus with the high risk of systemic embolization, atrial and pulmonary thrombectomy was immediately carried out. The cardiac thrombus with a length of $15 \mathrm{~cm}$ and a diameter of $10-15 \mathrm{~mm}$ was removed in toto (Panel C).

Surgery was uncomplicated and the patient made a rapid recovery so that he could be discharged on day 15 in a stable condition with the recommendation for oral anticoagulation therapy.

Bernhard Metzler, Thomas Schachner, Otmar Pachinger, Johannes Mair

\section{Conflict of interest}

The authors declare that there is no conflict of interest.

Key words: Patent foramen ovale, thrombus, echocardiography, pulmonary embolism.

Correspondence: Bernhard Metzler, MD, Clinical Division of Cardiology, Innsbruck Medical University, Anichstraße 35, A-6020 Innsbruck, Austria, E-mail: bernhard.metzler@uki.at 